gels than has been possible by studies of gels alone.

At constant temperature solutions of deoxyHb $\mathbf{S}$ remain liquid for long periods until they suddenly gel. The delay is exactly reproducible and is inversely proportional to high powers of the temperature and haemoglobin concentration (Proc. natn. Acad. Sci. U.S.A. 71, 4864; 1974). W. Eaton (Bethesda, Maryland) proposed to use this phenomenon for testing antisickling drugs. To make the disease significantly less severe, a drug would have to increase the delay 10-100-fold, equivalent to a 1.06-1.17-fold increase in the solubility of deoxyHb $\mathrm{S}$ or a 1.5-4-fold decrease in intracellular $\mathrm{Hb}$ concentration or a 4-7-fold increase in oxygen affinity. These are stiff requirements. E. R. Heuhns and M. Rosemeyer measured the antigelling properties of $\mathrm{Hb} \mathrm{A}, \mathrm{F}$ and $\mathrm{A}_{2}$ when added to $\mathrm{Hb} S$, and found that the antigelling effect rises in that order. This suggests that activation of the gene for $\mathrm{Hb} A$ would have a more potent antisickling effect than activation of the gene for $\mathrm{Hb}$ F.

\section{Cell studies}

Studies of whole normal and sickled cells were reviewed next. J. Hoffman (New Haven) discussed the distribution of diffusible ions and control of cell volume in normal erythrocytes, and the use of fluorescent dyes to study the intracellular milieu. Such studies are very relevant in view of the steep dependence of the gelling delay on haemoglobin concentration. M. Mohandas and N. Bessis (Paris) used anisotropy of laser light scattering to measure the deformability of red cells sheared between the concentric cylinders of a viscometer. This method could be used to measure the degree by which antisickling agents diminish the stiffening of deoxygenated sickle cells.

The physiology of the sickle erythrocyte is more complex than that of $\mathrm{Hb}$ A solutions. Recent technical advances are now allowing their oxygen equilibria to be measured. R. Bookchin (New York) found that the Bohr effect of sickle cells is steeper than that of normal ones and that 2,3-diphosphoglycerate, the intracellular oxygen regulator, increases gelation merely by lowering the oxygen affinity and not, as had been reported, by making deoxyHb A less soluble. R. Benesch and R. E. Benesch (New York) measured the oxygen affinity of $\mathrm{Hb} \mathrm{A}$ as a function of haemoglobin concentration and found a discontinuity at

M. F. Perutz is Director, MRC Laboratory of Molecular Biology, Cambridge, A. N. Schechter is at the Laboratory of Chemical Biology, NIAMDD, Bethesda and J. Rosa is Director, Unite et Recherche sur les Anemies, Hôpital Henri Moldur, Creteil. the point of gelation corresponding to the minimum gelling concentration. The concentration at which this discontinuity occurs could be used as a measure of the antigelling properties of drugs. If any drug inhibited sickling without raising the oxygen affinity of solutions which are too dilute to gel, then this would prove that it acted on the intermolecular contacts.

\section{Antigelling and antisickling agents}

Compounds being tested in vitro as antigelling or antisickling agents were discussed next. These may act by more than one mechanism, but can be classified according to their primary effects. The first class consists of modifiers of $\mathrm{Hb}$ A which raise the oxygen affinity. The prototype of such reagents was KNCO which proved too toxic for oral therapy. According to L. Kraus carbamylphosphate reacts with haemoglobin in the same way as cyanate, but the unreacted fraction may be less toxic as a result of enzymatic degradation. Imidoesters, both bifunctional dimethyladipimidate (B. Lubin, Oakland, California) and monofunctional methylacetimidate (T. Gabuzda, Philadelphia) decrease sickling by raising the oxygen affinity; so does cystamine which reacts with cysteines 93 $\beta$ (Y. Beuzard \& J. Rosa, Paris). Among disulphide reagents cystine dimethylester reacts with the same groups, increases the oxygen affinity and has antisickling properties demonstrable in single erythrocytes (D. L. Currell, Rome). The toxic nitrogen mustards react with histidine $2 \beta$ which is one of the diphosphoglycerate binding groups. (E. Roth, New York).

The second class consists of modifiers of intermolecular contacts in deoxyHb $S$ fibres. J. Manning (New York) has found that glyceraldehyde inhibits sickling, probably by reaction with a specific lysine not yet determined. It also acts on the membrane. Other compounds in this class interact noncovalently, such as the alkylureas studied by R. Nagel's group (New York) which are more potent inhibitors than urea itself. A. Schechter (Bethesda) has sought stereospecific amino acids or peptides that inhibit gelation and found that aromatic amino acids or peptides containing aromatic amino acids, but not the sickling peptide (residues 1-16 $\beta$ ), have antigelling properties. C. F. Poyart (Paris) found that methane, ethane, propane and butane increased the oxygen affinity by $10-15 \%$, propylene by $25 \%$ and butane by $50 \%$; these agents may also act on the intermolecular contacts. A. Cerami (New York) reported experiments with n-carboxyaspirindimethylester which reacts with an as yet undetermined group.

The final class of compounds dis- cussed were modifiers of the whole erythrocyte. G. Brewer (Ann Arbor) described clinical and laboratory studies of $\mathrm{Zn}$ acetate as an antisickling drug and C. Natta (New York) discussed DBA(3,4-dihydro-2,2-dimethyl2H-1-benzopyran-6-butyric acid).

Many of these compounds are too toxic for oral use and would have to be administered extracorporeally, by removing the red cells from the circulation, reacting them with the drugs, and putting them back again. S. Balcerzak (Columbus) has tested extracorporeal administration of cyanate to erythrocytes of dogs and two baboons and found no toxic effects in periods of up to one year. D. Diederich (Kansas) tried extracorporeal cyanate treatment on about 10 sickle cell patients and found a reduction in crises during treatment, as compared to before and after.

According to W. Rosse's (Durham, North Carolina) review of the American clinical trials of orally administered urea, alkali and cyanate, none of these reduced the severity or frequency of sickle cell crises, but the trials helped to establish reliable criteria for evaluating new therapies. At a final round table conference clinicians from West Africa and the USA dwelt on the great variability of the disease, and the absence of reliable information concerning its mortality, morbidity and clinical manifestations, especially in Africa and the Middle East. They were also concerned about the ethical problems of trying antisickling drugs on patients. They would not try them on children and felt that even adults might be better helped by conservative treatments.

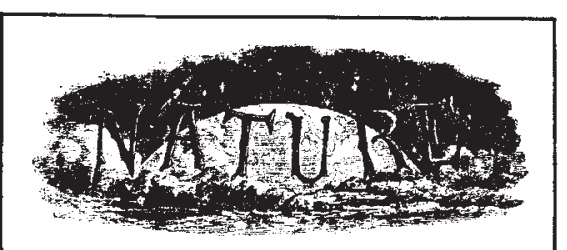

\section{A hundred years ago}

From the 16th to the 18th of August the city of Cartago in Costa Rica, in Central America, was visited by five distinct shocks of earthquake. No particular damage was caused. What is to be noticed in the meteorological result: that the weather was changed, being attended by heavy showers that flooded the rivers. In all earthquake countries it is believed the weather is affected by such phenomena, and it is desirable that observations should be recorded, showing the influence of earthquakes on weather. It has been alleged that the Comrie earthquakes in Perthshire came on after rains and floods of the river.

From Nature 3 October, 18, 600; 1878. 